\title{
Dark photon portal into mirror world
}

\author{
Abdaljalel Alizz ${ }^{*}$ \\ Novosibirsk State University, Novosibirsk 630 090, Russia \\ Z. K. Silagadze丹 \\ Budker Institute of Nuclear Physics and Novosibirsk State University, Novosibirsk 630 090, Russia
}

\begin{abstract}
Dark photons and mirror matter are well-motivated dark matter candidates. It is possible that both of them arose during the compactification and symmetry breaking scenario of the heterotic $E_{8} \times E_{8}$ string theory and are related to each other. In this case, dark photons can become a natural portal into the mirror world. Unfortunately, the expected magnitude of the induced interactions of ordinary matter with mirror matter is too small to be of phenomenological interest.
\end{abstract}

\section{INTRODUCTION}

There is overwhelming evidence for the existence of dark matter at all astrophysical length scales, from galactic to cosmological [1]. At the same time, the true nature and composition of dark matter remain unknown. All evidence for the existence of dark matter so far is based on its gravitational effects. Many proposed models of dark matter assume other non-gravitational very weak interactions between dark matter particles and ordinary matter. This motivates dark matter direct-detection experiments [2, 3]. The sensitivity of such experiments has improved tremendously. However, no clear experimental evidence for the existence of dark matter particles has been provided yet from these experiments.

A generic feature of string theory is the prediction of extra $U(1)$ gauge factors beyond the Standard Model group [4]. This fact makes dark photons well-motivated candidates for dark matter [5]. These dark photons can kinetically mix with the Standard Model photon [6] and thus lead to potentially observable effects in astrophysical and cosmological phenomena, as well as in laboratory experiments [5, 7].

Perhaps some comments about dark photons as dark matter candidate are appropriate here. Our usual intuition is that light vector bosons mediate forces, but cannot make up matter in our universe. However very light dark photons can challenge this intuition.

An interesting alternative to cold dark matter is the so-called fuzzy dark matter, consisting of very light bosons with extremely long de Broglie wavelengths [8, [9]. Inside galaxies, such dark matter can be modeled as a classical field and can lead to a variety of dark condensed matter physics phenomena [10].

If relic abundance of dark photon dark matter is produced by quantum fluctuations during inflation, then its energy density $\Omega_{B}$ relative to the critical density today is estimated to be [1]

$$
\Omega_{B}=\Omega_{D M} \sqrt{\frac{m_{B}}{6 \times 10^{-6} \mathrm{eV}}}\left(\frac{H_{I}}{10^{14} \mathrm{GeV}}\right)^{2},
$$

where the inflationary Hubble scale $H_{I} \leq 10^{14} \mathrm{GeV}$ is bounded by the absence of observation of primordial gravitational waves [10]. Therefore, in this scenario, dark photons from inflationary fluctuations can constitute the dominant part of the dark matter only if $m_{B} \geq \mu \mathrm{eV}$ [10]. However, other scenarios, when the dark photon dark matter abundance is generated by instabilities in a misaligned axion, operate over a wider range of dark photon masses down to $10^{-20} \mathrm{eV}[10]$.

Another well-motivated candidate for dark matter is mirror matter. The mirror partners of ordinary particles were first introduced by Lee and Yang in their famous paper 12] in an attempt to preserve the left-right symmetry of the world. Kobzarev, Okun and Pomeranchuk realized [13] that mirror particles could make up a hidden sector of the world, which communicates with the visible world mainly through gravity. The idea was rediscovered in the modern context of renormalizable gauge theories by Foot, Lew and Volkas 14] and revived in the context of neutrino oscillations in both mirror-symmetric [15] and mirror-asymmetric [16] forms. For recent reviews of the mirror matter theory and related references, see, for example, [17 20].

*Electronic address: abdaljalel90@gmail.com

${ }^{\dagger}$ Electronic address: Z.K.Silagadze@inp.nsk.su 
Mirror matter, like dark photons, can also be motivated (with some caveats) by string theory. One of the heterotic string theories, which makes it possible to construct a coherent quantum theory that unifies all interactions, including gravity, is based on the group $E_{8} \times E_{8}[23,24]$. The second $E_{8}$ after compactification can lead to the existence of shadow matter, which interacts only gravitationally with ordinary matter [25]. Mirror matter is a special case of shadow matter when the symmetry breaking patterns of the second $E_{8}$ exactly mirror the patterns of the first $E_{8}$, which leads to an exciting possibility of a mirror world with invisible stars, planets and galaxies [13, 26].

Some of the caveats mentioned above are as follows. In the $E_{8} \times E_{8}$ heterotic string model we have an exact symmetry between two $E_{8}$-s at the stringy scale when the basic constituents of the universe are strings living in ten-dimensional space-time (as dictated by anomaly cancellation). Then universe cools and the extra six dimensions compactify in the spirit of Kaluza-Klein theory, so that the space-time becomes $M_{4} \times K_{6}$, where $M_{4}$ is a familiar 4-dimensional space-time and $K_{6}$ is some compact 6-dimensional manifold. Usually one demands an unbroken $N=1$ supersymmetry in four dimensions and in this case the natural choice for $K_{6}$ is a Calabi-Yau manifold [27].

We emphasize that nothing in the theory itself requires such compactification. This is just a phenomenological assumption, very popular around the turn of the millennium (but perhaps less popular today due to the lack of experimental evidence for supersymmetry).

Even if one assumes this particular type of compactification, the number of possible phenomenologically viable compactifications are huge. For complete intersection Calabi-Yau threefolds the number of string theory standard models is estimated to be $10^{23}$ (a mole of standard models in Tristan Hübsch's apt characterization), and for the class of Calabi-Yau hypersurfaces in toric varieties, the estimated number of standard models is truly terrifying $10^{723}$ [28]. It is safe to say that it is currently unclear which path leads from string theory to low-energy physics of the Standard Model, and it is also unclear whether string theory actually describes nature.

The standard Calabi-Yau compactification breaks the mirror symmetry between two $E_{8}$-s because the $S U(3)$ holonomy group of the Calabi-Yau manifold is chosen in one of the $E_{8}$-factors breaking it to $E_{6}$, while the second $E_{8}$ remains unbroken.

One might think that an alternative possibility is to embed the $\mathrm{SU}(3)$ holonomy in a diagonal subgroup of $E_{8} \times E_{8}$ and in this way maintain the symmetry between the visible and shadow matters. Unfortunately, such Calabi-Yau spaces have not been constructed [29].

Therefore, in what follows, for definiteness, we will focus on mirror matter, but the scenario considered is actually applicable for a wider class of shadow matter theories, which do not necessarily assume exact mirror symmetry between ordinary and shadow particles.

Since both dark photons and mirror matter may have their origins in string theory, it can be assumed that they are related in some way ${ }^{1}$. In this short note, we present the simplest model of this type: the visible and shadow sectors, connected only (except gravity) by dark photon messengers.

Although our initial motivation was a hint from string theory, we do not really need this hypothesis for what follows. Equally successfully can one motivate such a picture from the $E_{6} \times E_{6}$ grand unification theory with symmetric or asymmetric symmetry breaking patterns of two $E_{6}$-s $[31]$.

\section{MIRROR WORLD WITH DARK PHOTONS}

Calabi-Yau compactifications of the heterotic $E_{8} \times E_{8}$ superstring model naturally leads to the gauge group $E_{6}$ in the visible sector [26, 32]. Further symmetry breaking patterns such as $E_{6} \rightarrow S O(10) \times U(1)$ or $E_{6} \rightarrow S U(5) \times S U(2) \times U(1)$ can introduce the gauge group $U(1)$ of dark photons into play ${ }^{2}$.

It is usually assumed that after Calabi-Yau compactification, the gauge group in the hidden sector is different from the visible sector gauge group, since, as noted in [25], a fully symmetric hidden sector contradicts observations, in particular Big Bang nucleosynthesis constraints. However, the limitations from the Big Bang nucleosynthesis can be avoided if the mirror sector has a lower temperature than the ordinary one, and an inflationary scenario can be envisaged that could explain the different initial temperatures of the two sectors [20]. As a result, although the microphysics of these two sectors are identical, their macroscopic properties in relation to the most important epochs, such as baryogenesis, nucleosynthesis, etc., will be completely different [20].

Therefore, we assume that it is possible to maintain either an exact mirror symmetry or its broken version between the visible and hidden sectors, starting from the scale of the $E_{6} \times E_{6}$ grand unification and up to the electroweak

\footnotetext{
${ }^{1}$ In addition to string theory, both mirror matter and dark photons were motivated in a five-dimensional unification of gravitation and electromagnetism using a degenerate metric [30].

${ }^{2} E_{8} \times E_{8}$ superstring phenomenology, including shadow world was discussed, for example, in [33].
} 
scale. Then during, say, $E_{6} \times E_{6} \rightarrow(S O(10) \times U(1)) \times(S O(10) \times U(1))$ symmetry breaking stage the two $U(1)$-s of the dark photon and mirror dark photon can get mixed, for example, through the Higgs portal [34].

These considerations prompt us to consider a scenario when mirror matter (or its shadow analogue) predominates in dark matter, and dark photons play merely the role of a portal between the visible and hidden sectors. We describe this situation by an effective Lagrangian density

$$
\mathcal{L}=L+\tilde{L}-\frac{\epsilon_{2}}{2} F_{b \mu \nu} \tilde{F}^{b \mu \nu}-e_{a} J_{\mu} A^{a \mu}-e_{a} \tilde{J}_{\mu} \tilde{A}^{a \mu}
$$

where tilde denotes mirror fields, $J_{\mu}$ and $\tilde{J}_{\mu}$ are ordinary and mirror electric currents, and

$$
\begin{aligned}
L & =-\frac{1}{4} F_{a \mu \nu} F^{a \mu \nu}-\frac{1}{4} F_{b \mu \nu} F^{b \mu \nu}+\frac{1}{2} m_{b}^{2} A_{b \mu} A^{b \mu}-\frac{\epsilon_{1}}{2} F_{a \mu \nu} F^{b \mu \nu}, \\
\tilde{L} & =-\frac{1}{4} \tilde{F}_{a \mu \nu} \tilde{F}^{a \mu \nu}-\frac{1}{4} \tilde{F}_{b \mu \nu} \tilde{F}^{b \mu \nu}+\frac{1}{2} m_{b}^{2} \tilde{A}_{b \mu} \tilde{A}^{b \mu}-\frac{\epsilon_{1}}{2} \tilde{F}_{a \mu \nu} \tilde{F}^{b \mu \nu} .
\end{aligned}
$$

The dark photon masses can arise (in each sector), for example, by Stückelberg mechanism [35], or by the usual Higgs mechanism.

The Lagrangian (2) can be diagonalized in three steps. First, we introduce the fields $A_{\mu}$ and $B_{\mu}^{\prime}$ along their mirror counterparts via

$$
\begin{aligned}
& A_{a \mu}=A_{\mu}-\frac{\epsilon_{1}}{\sqrt{1-\epsilon_{1}^{2}}} B_{\mu}^{\prime}, \quad A_{b \mu}=\frac{1}{\sqrt{1-\epsilon_{1}^{2}}} B_{\mu}^{\prime}, \\
& \tilde{A}_{a \mu}=\tilde{A}_{\mu}-\frac{\epsilon_{1}}{\sqrt{1-\epsilon_{1}^{2}}} \tilde{B}_{\mu}^{\prime}, \quad \tilde{A}_{b \mu}=\frac{1}{\sqrt{1-\epsilon_{1}^{2}}} \tilde{B}_{\mu}^{\prime} .
\end{aligned}
$$

In terms of these fields, Lagrangians (3) take the diagonal forms

$$
\begin{aligned}
& L=-\frac{1}{4} F_{\mu \nu} F^{\mu \nu}-\frac{1}{4} B_{\mu \nu}^{\prime} B^{\prime \mu \nu}+\frac{1}{2} \frac{m_{b}^{2}}{1-\epsilon_{1}^{2}} B_{\mu}^{\prime} B^{\prime \mu}, \\
& \tilde{L}=-\frac{1}{4} \tilde{F}_{\mu \nu} \tilde{F}^{\mu \nu}-\frac{1}{4} \tilde{B}_{\mu \nu}^{\prime} \tilde{B}^{\prime \mu \nu}+\frac{1}{2} \frac{m_{b}^{2}}{1-\epsilon_{1}^{2}} \tilde{B}_{\mu}^{\prime} \tilde{B}^{\prime \mu} .
\end{aligned}
$$

However, visible and hidden gauge fields still remain kinematically intermixed thanks to the $-\frac{\epsilon_{2}}{2\left(1-\epsilon_{1}^{2}\right)} B_{\mu \nu}^{\prime} \tilde{B}^{\prime \mu \nu}$ term. This term can be diagonalized by the transformation

$$
B_{\mu}^{\prime}=\frac{1}{\sqrt{2}}\left(B_{\mu}^{\prime \prime}+\tilde{B}_{\mu}^{\prime \prime}\right), \quad \tilde{B}_{\mu}^{\prime}=\frac{1}{\sqrt{2}}\left(B_{\mu}^{\prime \prime}-\tilde{B}_{\mu}^{\prime \prime}\right) .
$$

Being an orthogonal transformation, (6) does not spoil the diagonality of the sum of Lagrangians (5). However, we end up with incorrect coefficients of the kinetic $B_{\mu \nu}^{\prime \prime} B^{\prime \prime \mu \nu}$ and $\tilde{B}_{\mu \nu}^{\prime \prime} \tilde{B}^{\prime \prime \mu \nu}$ terms. To restore the correct normalization of these terms, we rescale the fields:

$$
B_{\mu}^{\prime \prime}=\sqrt{\frac{1-\epsilon_{1}^{2}}{1-\epsilon_{1}^{2}+\epsilon_{2}}} B_{\mu}, \quad \tilde{B}_{\mu}^{\prime \prime}=\sqrt{\frac{1-\epsilon_{1}^{2}}{1-\epsilon_{1}^{2}-\epsilon_{2}}} \tilde{B}_{\mu} .
$$

Finally, in terms of mass-eigenstate physical fields (ordinary photon $A_{\mu}$, mirror photon $\tilde{A}_{\mu}$, dark photon $B_{\mu}$ and mirror dark photon $\tilde{B}_{\mu}$ ) our original Lagrangian density (2) takes the form

$$
\mathcal{L}=-\frac{1}{4} F_{\mu \nu} F^{\mu \nu}-\frac{1}{4} B_{\mu \nu} B^{\mu \nu}-\frac{1}{4} \tilde{F}_{\mu \nu} \tilde{F}^{\mu \nu}-\frac{1}{4} \tilde{B}_{\mu \nu} \tilde{B}^{\mu \nu}+\frac{1}{2} \mu^{2} B_{\mu} B^{\mu}+\frac{1}{2} \tilde{\mu}^{2} \tilde{B}_{\mu} \tilde{B}^{\mu}+\mathcal{L}_{\text {int }} .
$$

Here, as usual, $F_{\mu \nu}=\partial_{\mu} A_{\nu}-\partial_{\nu} A_{\mu}, B_{\mu \nu}=\partial_{\mu} B_{\nu}-\partial_{\nu} B_{\mu}$, and

$$
\mu^{2}=\frac{m_{b}^{2}}{1-\epsilon_{1}^{2}+\epsilon_{2}}, \quad \tilde{\mu}^{2}=\frac{m_{b}^{2}}{1-\epsilon_{1}^{2}-\epsilon_{2}} .
$$

The most interesting is the interaction term

$$
\mathcal{L}_{\mathrm{int}}=-e_{a} J_{\mu}\left[A^{\mu}-\frac{\epsilon_{1}}{\sqrt{2}}\left(\frac{B^{\mu}}{\sqrt{1-\epsilon_{1}^{2}+\epsilon_{2}}}+\frac{\tilde{B}^{\mu}}{\sqrt{1-\epsilon_{1}^{2}-\epsilon_{2}}}\right)\right]-e_{a} \tilde{J}_{\mu}\left[\tilde{A}^{\mu}-\frac{\epsilon_{1}}{\sqrt{2}}\left(\frac{B^{\mu}}{\sqrt{1-\epsilon_{1}^{2}+\epsilon_{2}}}-\frac{\tilde{B}^{\mu}}{\sqrt{1-\epsilon_{1}^{2}-\epsilon_{2}}}\right)\right] .
$$


As we can see, the effect of $\sim \epsilon_{2}$ mixing term in (2) is twofold: it removes the degeneracy between dark photon and mirror dark photon, resulting in mass splitting

$$
\mu^{2}-\tilde{\mu}^{2}=-\frac{2 \epsilon_{2} m_{b}^{2}}{\left(1-\epsilon_{1}^{2}\right)^{2}-\epsilon_{2}^{2}} \approx-2 \epsilon_{2} m_{b}^{2}
$$

and interconnects the visible and hidden sectors (if $\epsilon_{2}=0$, the interconnection implied by (10) is unphysical and can be rotated away by an orthogonal transformation of the physical fields).

\section{CONCLUDING REMARKS}

Previously, several different "portals" were considered that connect the ordinary and mirror worlds: photon portal (photon-mirror photon oscillations) [36, 37], neutrino portal [15, 16, 38, 39], Higgs portal [40 42], axion portal [43 45], neutron portal (neutron-mirror neutron oscillations) $[46]^{3}$. Even in the absence of all these portals, the visible and mirror sectors become interconnected (albeit very weakly) by quantum gravity effects [51].

The dark photon portal proposed in this note is, in our opinion, very natural. Combining two dark entities is also helpful in explaining the dark matter mystery. For light dark photons to be the dominant part of dark matter, it is necessary to identify a well-motivated mechanism for their production. Although some production mechanisms have been proposed (misalignment mechanism, production due to fluctuations of the metric during inflation of the early universe, temperature-dependent instabilities in the hidden-sector (pseudo)scalar field coupled to a dark photon), they are not without difficulties 52 .

In the case of mirror matter, there are no such difficulties, and mirror baryons could easily provide the dominant component of dark matter in the universe [20]. For example, interactions leading to mixing of neutrinos of two sectors can co-generate both types of matter [21], naturally leading to $\tilde{\Omega}_{B}>\Omega_{B}[22]$.

Dark photons and mirror matter can combine to form a multi-component dark matter with properties as diverse as ordinary matter in the universe, and perhaps astrophysical observations do indicate diverse behavior of dark matter in galaxy cluster collisions [53].

Dark photons and mirror matter help each other to stay hidden from direct experimental searches, such as experiments like XENON1T [54], or in the search for invisible decays of positronium [55]. Indeed, it is clear from (10) that positronium can oscillate into mirror positronium through $B_{\mu}$ or $\tilde{B}_{\mu}$ exchange. However, the amplitude of this transition is proportional to (assuming ultralight dark photons, $m_{b} \ll m_{e}$ )

$$
\mathcal{A}(P s \rightarrow \widetilde{P s}) \sim\left(\frac{\epsilon_{1}}{\sqrt{2}} \frac{1}{\sqrt{1-\epsilon_{1}^{2}+\epsilon_{2}}}\right)^{2}-\left(\frac{\epsilon_{1}}{\sqrt{2}} \frac{1}{\sqrt{1-\epsilon_{1}^{2}-\epsilon_{2}}}\right)^{2}=-\frac{\epsilon_{1}^{2} \epsilon_{2}}{\left(1-\epsilon_{1}^{2}\right)^{2}-\epsilon_{2}^{2}} \approx-\epsilon_{1}^{2} \epsilon_{2} .
$$

Bounds on $\epsilon_{1}$ from the dark photon searches are quite tight [56]. It is probably safe to assume that $\left|\epsilon_{1}\right|<10^{-7}$. Then (12) is unobservable small for any reasonable value of $\epsilon_{2}$.

As we are playing with the possibility that at the low energy limit the only messengers between the ordinary and hidden sectors are their dark photons, our effective Lagrangian (3) does not contain photon-mirror photon mixing. Significant amount of such mixing would imply rather rich phenomenology and can provide a straightforward portal for the dark matter detection [57 60]. However, such mixing is severely limited experimentally [55], and from the Big Bang nucleosynthesis, as well as from the cosmic microwave background measurements and large scale structure considerations [61, 62.

For the Lagrangian (3), the photon-mirror photon mixing effectively is induced due to the dark photon-mirror dark photon mixing. However, this effective mixing is naturally small, of the order of $\epsilon_{1}^{2} \epsilon_{2}$, and constraints on $\epsilon_{1}$ from the dark photon searches imply that the mixing is expected to be far below the limits mentioned above.

The situation is different for asymmetric mirror matter scenario, when the mirror photon-mirror dark photon mixing parameter $\tilde{\epsilon}_{1}$ not necessarily equals to $\epsilon_{1}$. Since the mirror photon-mirror dark photon and dark photon-mirror dark photon kinetic mixings are not tightly constrained, the induced photon-mirror photon mixing parameter $\epsilon_{1} \tilde{\epsilon}_{1} \epsilon_{2}$ can have a phenomenologically interesting magnitude of about $10^{-10}$.

\footnotetext{
${ }^{3}$ It was stated in [47] that the internal heating of a neutron star severely constraints the neutron portal. However, the internal heating of a neutron star was discussed earlier in [48], and it was concluded that the constraints are not at all so severe: they are comparable to the direct experimental bounds [49, 50] and thus still allow neutron oscillations much faster than the neutron decay.
} 
However, for symmetric mirror matter, it will be impossible to solve the small-scale structure puzzles with dissipative mirror dark matter as considered in [63], since such a solution requires $\epsilon_{1}^{2} \epsilon_{2} \sim 2 \cdot 10^{-10}[63]^{4}$. However, this conclusion is correct only if there are no stable dark particles charged under the gauge group of dark photons, which can play the role of dissipative dark matter in solving problems of small-scale structure if $\epsilon_{1} \sim 10^{-9}$ is not that small [65, 66].

Contrariwise, with mirror matter at hand, there is no need to assume that dark photons represent a significant fraction of galactic dark matter. Therefore, dark photon direct-search experiments can only rely on intense photon sources such as the Sun.

If the dark photon portal is a dominant mechanism connecting visible and mirror hidden sectors, and if $\epsilon_{1}$ and $\epsilon_{2}$ are both too small, then unfortunately experiments to directly search for mirror dark matter will have no more chance of success than finding black cat in a dark room.

However, we do not want to become victims of the "Pygmalion Syndrome" 67], and confuse the real world with what we have imagined here. Mirror dark matter search experiments are very important, since only in this way we can check our models of reality. The pessimistic expectation for the results of such experiments is just a testable prediction of the particular model discussed in this article.

\section{Acknowledgments}

We are grateful to Maxim Khlopov, Trevor Searight, Nirmal Raj, Sunny Vagnozzi, and Robert McGehee for useful correspondence. Constructive criticism from an anonymous reviewer helped improve the presentation of the manuscript. The work is supported by the Ministry of Education and Science of the Russian Federation.

\section{Appendix: Alternative method of diagonalization}

The Referee suggested the following alternative method of diagonalization. The method is not simpler than the one given in the main text, but nevertheless it is useful for checking calculations, and since its ideology is common when considering particle mixing.

Let's write the Lagrangian (2) in matrix form:

$$
\mathcal{L}=-\frac{1}{4} \mathbf{F}_{\mu \nu}^{T} \mathbf{K} \mathbf{F}^{\mu \nu}+\frac{1}{2} \mathbf{A}_{\mu}^{T} \mathbf{M} \mathbf{A}^{\mu}-e_{a} \mathbf{J}_{\mu}^{T} \mathbf{A}^{\mu}
$$

where

$$
\mathbf{K}=\left(\begin{array}{cccc}
1 & \epsilon_{1} & 0 & 0 \\
\epsilon_{1} & 1 & 0 & \epsilon_{2} \\
0 & 0 & 1 & \epsilon_{1} \\
0 & \epsilon_{2} & \epsilon_{1} & 1
\end{array}\right), \mathbf{M}=\left(\begin{array}{cccc}
0 & 0 & 0 & 0 \\
0 & m_{b}^{2} & 0 & 0 \\
0 & 0 & 0 & 0 \\
0 & 0 & 0 & m_{b}^{2}
\end{array}\right)
$$

and

$$
\mathbf{F}^{\mu \nu}=\left(\begin{array}{c}
F_{a}^{\mu \nu} \\
F_{b}^{\mu \nu} \\
\tilde{F}_{a}^{\mu \nu} \\
\tilde{F}_{b}^{\mu \nu}
\end{array}\right), \mathbf{A}^{\mu}=\left(\begin{array}{c}
A_{a}^{\mu} \\
A_{b}^{\mu} \\
\tilde{A}_{a}^{\mu} \\
\tilde{A}_{b}^{\mu}
\end{array}\right), \mathbf{J}_{\mu}=\left(\begin{array}{c}
J_{\mu} \\
0 \\
\tilde{J}_{\mu} \\
0
\end{array}\right)
$$

The kinetic matrix $\mathbf{K}$ is reduced to the identity matrix by a linear transformation $\mathbf{A}^{\mu}=\mathbf{P} \mathbf{A}^{\prime \mu}$, where

$$
\mathbf{P}=\frac{1}{\sqrt{2}}\left(\begin{array}{cccc}
\frac{\cos \theta}{\sqrt{\lambda_{1}}} & \frac{\sin \theta}{\sqrt{\lambda_{2}}} & \frac{-\cos \theta}{\sqrt{\lambda_{3}}} & \frac{\sin \theta}{\sqrt{\lambda_{4}}} \\
\frac{-\sin \theta}{\sqrt{\lambda_{1}}} & \frac{\cos \theta}{\sqrt{\lambda_{2}}} & \frac{-\sin \theta}{\sqrt{\lambda_{3}}} & \frac{-\cos \theta}{\sqrt{\lambda_{4}}} \\
\frac{\cos \theta}{\sqrt{\lambda_{1}}} & \frac{\sin \theta}{\sqrt{\lambda_{2}}} & \frac{\cos \theta}{\sqrt{\lambda_{3}}} & \frac{-\sin \theta}{\sqrt{\lambda_{4}}} \\
\frac{-\sin \theta}{\sqrt{\lambda_{1}}} & \frac{\cos \theta}{\sqrt{\lambda_{2}}} & \frac{\sin \theta}{\sqrt{\lambda_{3}}} & \frac{\cos \theta}{\sqrt{\lambda_{4}}}
\end{array}\right)=\mathbf{S}\left(\begin{array}{cccc}
\frac{1}{\sqrt{\lambda_{1}}} & 0 & 0 & 0 \\
0 & \frac{1}{\sqrt{\lambda_{2}}} & 0 & 0 \\
0 & 0 & \frac{1}{\sqrt{\lambda_{3}}} & 0 \\
0 & 0 & 0 & \frac{1}{\sqrt{\lambda_{4}}}
\end{array}\right)
$$

\footnotetext{
${ }^{4}$ Perhaps, the small-scale structure puzzle is not a puzzle at all for the mirror matter model, although it may be a problem for generic dissipative dark matter matter. It was shown in [64] that the scale of damping of mirror structures depends on the temperature ratio between the mirror and ordinary sectors, and for small enough ratio of about 0.1-0.2, small scales are suppressed.
} 
$\mathbf{S}$ is an orthogonal matrix,

$$
\begin{array}{ll}
\lambda_{1}=1+\frac{1}{2}\left(\epsilon_{2}-\sqrt{4 \epsilon_{1}^{2}+\epsilon_{2}^{2}}\right), & \lambda_{2}=1+\frac{1}{2}\left(\epsilon_{2}+\sqrt{4 \epsilon_{1}^{2}+\epsilon_{2}^{2}}\right), \\
\lambda_{3}=1+\frac{1}{2}\left(-\epsilon_{2}+\sqrt{4 \epsilon_{1}^{2}+\epsilon_{2}^{2}}\right), & \lambda_{4}=1-\frac{1}{2}\left(\epsilon_{2}+\sqrt{4 \epsilon_{1}^{2}+\epsilon_{2}^{2}}\right)
\end{array}
$$

are eigenvalues of the matrix $\mathbf{K}$, and

$$
\tan 2 \theta=\frac{2 \epsilon_{1}}{\epsilon_{2}}, \quad \cos 2 \theta=\frac{\epsilon_{2}}{\sqrt{4 \epsilon_{1}^{2}+\epsilon_{2}^{2}}}
$$

Note that

$$
\mathbf{S}=\left(\begin{array}{cccc}
\frac{1}{\sqrt{2}} & 0 & \frac{1}{\sqrt{2}} & 0 \\
0 & \frac{1}{\sqrt{2}} & 0 & \frac{1}{\sqrt{2}} \\
\frac{1}{\sqrt{2}} & 0 & \frac{-1}{\sqrt{2}} & 0 \\
0 & \frac{1}{\sqrt{2}} & 0 & \frac{-1}{\sqrt{2}}
\end{array}\right)\left(\begin{array}{rrrr}
\cos \theta & \sin \theta & 0 & 0 \\
-\sin \theta & \cos \theta & 0 & 0 \\
0 & 0 & -\cos \theta & \sin \theta \\
0 & 0 & -\sin \theta & -\cos \theta
\end{array}\right)
$$

The mass matrix $\mathbf{M}^{\prime}=\mathbf{P}^{T} \mathbf{M P}$ ceases to be diagonal after the transformation $\mathbf{P}$ :

$$
\mathbf{M}^{\prime}=m_{b}^{2}\left(\begin{array}{cccc}
\frac{\sin ^{2} \theta}{\lambda_{1}} & -\frac{\sin \theta \cos \theta}{\sqrt{\lambda_{1} \lambda_{2}}} & 0 & 0 \\
-\frac{\sin \theta \cos \theta}{\sqrt{\lambda_{1} \lambda_{2}}} & \frac{\cos ^{2} \theta}{\lambda_{2}} & 0 & 0 \\
0 & 0 & \frac{\sin ^{2} \theta}{\lambda_{3}} & \frac{\sin \theta \cos \theta}{\sqrt{\lambda_{3} \lambda_{4}}} \\
0 & 0 & \frac{\sin \theta \cos \theta}{\sqrt{\lambda_{3} \lambda_{4}}} & \frac{\cos ^{2} \theta}{\lambda_{4}}
\end{array}\right)
$$

The upper and lower quarters of this matrix have zero determinants. Therefore, the eigenvalues of $\mathbf{M}^{\prime}$ are $0, \mu^{2}, 0, \tilde{\mu}^{2}$, where

$$
\begin{aligned}
& \mu^{2}=m_{b}^{2}\left(\frac{\sin ^{2} \theta}{\lambda_{1}}+\frac{\cos ^{2} \theta}{\lambda_{2}}\right)=\frac{m_{b}^{2}}{2 \lambda_{1} \lambda_{2}}\left[\lambda_{1}+\lambda_{2}+\left(\lambda_{1}-\lambda_{2}\right) \cos 2 \theta\right], \\
& \tilde{\mu}^{2}=m_{b}^{2}\left(\frac{\sin ^{2} \theta}{\lambda_{3}}+\frac{\cos ^{2} \theta}{\lambda_{4}}\right)=\frac{m_{b}^{2}}{2 \lambda_{3} \lambda_{4}}\left[\lambda_{3}+\lambda_{4}+\left(\lambda_{3}-\lambda_{4}\right) \cos 2 \theta\right] .
\end{aligned}
$$

Substituting $\lambda$-s from (A.5) and $\cos 2 \theta$ from (A.6), we recover the relations (9).

The mass matrix $\mathbf{M}^{\prime}$ is diagonalized by an orthogonal transformation with the matrix (note that, as follows from (A.5) and (A.6), $\lambda_{1} \cos ^{2} \theta+\lambda_{2} \sin ^{2} \theta=1$ and $\lambda_{3} \cos ^{2} \theta+\lambda_{4} \sin ^{2} \theta=1$ )

$$
\mathbf{S}_{1}=\left(\begin{array}{rrrr}
\sqrt{\lambda_{1}} \cos \theta & -\sqrt{\lambda_{2}} \sin \theta & 0 & 0 \\
\sqrt{\lambda_{2}} \sin \theta & \sqrt{\lambda_{1}} \cos \theta & 0 & 0 \\
0 & 0 & -\sqrt{\lambda_{3}} \cos \theta & -\sqrt{\lambda_{4}} \sin \theta \\
0 & 0 & \sqrt{\lambda_{4}} \sin \theta & -\sqrt{\lambda_{3}} \cos \theta
\end{array}\right) .
$$

The transformation $\mathbf{S}_{\mathbf{1}}$ does not spoil the canonical form of kinetic terms, since it is orthogonal.

Photon and mirror photon are degenerate in mass (both massless), therefore, the corresponding combinations of fields are not uniquely determined (only up to an orthogonal transformation between the corresponding fields). In (10) we choose the "flavor" basis for them (the photon is connected only to the ordinary electric current, and the mirror photon is connected only to the mirror electric current). To get the same identification of fields, we need one more orthogonal transformation

$$
\mathbf{S}_{2}=\left(\begin{array}{cccc}
\frac{1}{\sqrt{2}} & 0 & \frac{1}{\sqrt{2}} & 0 \\
0 & 1 & 0 & 0 \\
\frac{1}{\sqrt{2}} & 0 & \frac{-1}{\sqrt{2}} & 0 \\
0 & 0 & 0 & 1
\end{array}\right)
$$

In total, the transformation $\mathbf{A}^{\mu}=\mathbf{P S}_{\mathbf{1}} \mathbf{S}_{\mathbf{2}} \mathbf{B}^{\mu}$, where

$$
\mathbf{P S}_{\mathbf{1}} \mathbf{S}_{2}=\left(\begin{array}{cccc}
1 & \frac{-\epsilon_{1}}{\sqrt{2\left(1+\epsilon_{2}-\epsilon_{1}^{2}\right)}} & 0 & \frac{-\epsilon_{1}}{\sqrt{2\left(1-\epsilon_{2}-\epsilon_{1}^{2}\right)}} \\
0 & \frac{1}{\sqrt{2\left(1+\epsilon_{2}-\epsilon_{1}^{2}\right)}} & 0 & \frac{1}{\sqrt{2\left(1-\epsilon_{2}-\epsilon_{1}^{2}\right)}} \\
0 & \frac{-\epsilon_{1}}{\sqrt{2\left(1+\epsilon_{2}-\epsilon_{1}^{2}\right)}} & 1 & \frac{\epsilon_{1}}{\sqrt{2\left(1-\epsilon_{2}-\epsilon_{1}^{2}\right)}} \\
0 & \frac{1}{\sqrt{2\left(1+\epsilon_{2}-\epsilon_{1}^{2}\right)}} & 0 & \frac{1}{\sqrt{2\left(1-\epsilon_{2}-\epsilon_{1}^{2}\right)}}
\end{array}\right), \quad \mathbf{B}^{\mu}=\left(\begin{array}{c}
A^{\mu} \\
B^{\mu} \\
\tilde{A}^{\mu} \\
\tilde{B}^{\mu}
\end{array}\right)
$$


brings the Lagrangian (A.1) exactly in the form described by equations (8), (9) and (10).

[1] G. Bertone, D. Hooper and J. Silk, Particle dark matter: Evidence, candidates and constraints, Phys. Rept. 405, 279-390 (2005). https://doi.org/10.1016/j.physrep.2004.08.031

[2] J. L. Feng, Dark Matter Candidates from Particle Physics and Methods of Detection, Ann. Rev. Astron. Astrophys. 48, 495-545 (2010). https://doi.org/10.1146/annurev-astro-082708-101659

[3] T. Marrodán Undagoitia and L. Rauch, Dark matter direct-detection experiments, J. Phys. G 43, 013001 (2016). https://doi.org/10.1088/0954-3899/43/1/013001

[4] M. Goodsell, J. Jaeckel, J. Redondo and A. Ringwald, Naturally Light Hidden Photons in LARGE Volume String Compactifications, JHEP 11 (2009), 027. https://doi.org/10.1088/1126-6708/2009/11/027

[5] M. Fabbrichesi, E. Gabrielli and G. Lanfranchi, The Physics of the Dark Photon: A Primer (Springer Nature: Cham, 2021). https://doi.org/10.1007/978-3-030-62519-1

[6] B. Holdom, Two U(1)'s and Epsilon Charge Shifts, Phys. Lett. B 166 (1986), 196-198 (1986). https://doi.org/10.1016/0370-2693(86)91377-8

[7] A. Filippi and M. De Napoli, Searching in the dark: the hunt for the dark photon, Rev. Phys. 5, 100042 (2020). https://doi.org/10.1016/j.revip.2020.100042

[8] W. Hu, R. Barkana and A. Gruzinov, Cold and fuzzy dark matter, Phys. Rev. Lett. 85, 1158-1161 (2000). https://doi.org/10.1103/PhysRevLett.85.1158

[9] L. Hui, J. P. Ostriker, S. Tremaine and E. Witten, Ultralight scalars as cosmological dark matter, Phys. Rev. D 95, 043541 (2017). https://doi.org/10.1103/PhysRevD.95.043541

[10] P. Agrawal, N. Kitajima, M. Reece, T. Sekiguchi and F. Takahashi, Relic Abundance of Dark Photon Dark Matter, Phys. Lett. B 801, 135136 (2020). https://doi.org/10.1016/j.physletb.2019.135136

[11] P. W. Graham, J. Mardon and S. Rajendran, Vector Dark Matter from Inflationary Fluctuations, Phys. Rev. D 93, 103520 (2016). https://doi.org/10.1103/PhysRevD.93.103520

[12] T. D. Lee and C. N. Yang, Question of Parity Conservation in Weak Interactions, Phys. Rev. 104, 254-258 (1956). https://doi.org/10.1103/PhysRev.104.254

[13] I. Y. Kobzarev, L. B. Okun and I. Y. Pomeranchuk, On the possibility of experimental observation of mirror particles, Sov. J. Nucl. Phys. 3, 837-841 (1966).

[14] R. Foot, H. Lew and R. R. Volkas, A Model with fundamental improper space-time symmetries, Phys. Lett. B 272, 67-70 (1991). https://doi.org/10.1016/0370-2693(91)91013-L

[15] R. Foot and R. R. Volkas, Neutrino physics and the mirror world: How exact parity symmetry explains the solar neutrino deficit, the atmospheric neutrino anomaly and the LSND experiment, Phys. Rev. D 52, 6595-6606 (1995). https://doi.org/10.1103/PhysRevD.52.6595

[16] Z. G. Berezhiani and R. N. Mohapatra, Reconciling present neutrino puzzles: Sterile neutrinos as mirror neutrinos, Phys. Rev. D 52, 6607-6611 (1995). https://doi.org/10.1103/PhysRevD.52.6607

[17] L. B. Okun, Mirror particles and mirror matter: 50 years of speculations and search, Phys. Usp. 50, $380-389$ (2007). https://doi.org/10.1070/PU2007v050n04ABEH006227

[18] R. Foot, Mirror dark matter: Cosmology, galaxy structure and direct detection, Int. J. Mod. Phys. A 29, 1430013 (2014). https://doi.org/10.1142/S0217751X14300130

[19] Z. Berezhiani, Mirror world and its cosmological consequences, Int. J. Mod. Phys. A 19, 3775-3806 (2004). https://doi.org/10.1142/S0217751X04020075

[20] Z. Berezhiani, D. Comelli and F. L. Villante, The Early mirror universe: Inflation, baryogenesis, nucleosynthesis and dark matter, Phys. Lett. B 503, 362-375 (2001). https://doi.org/10.1016/S0370-2693(01)00217-9

[21] L. Bento and Z. Berezhiani, Leptogenesis via collisions: The Lepton number leaking to the hidden sector, Phys. Rev. Lett. 87, 231304 (2001). https://doi.org/10.1103/PhysRevLett.87.231304

[22] Z. Berezhiani, Unified picture of ordinary and dark matter genesis, Eur. Phys. J. ST 163, 271-289 (2008). https://doi.org/10.1140/epjst/e2008-00824-6

[23] D. J. Gross, J. A. Harvey, E. J. Martinec and R. Rohm, The Heterotic String, Phys. Rev. Lett. 54, 502-505 (1985). https://doi.org/10.1103/PhysRevLett.54.502

[24] D. J. Gross, J. A. Harvey, E. J. Martinec and R. Rohm, Heterotic String Theory. 1. The Free Heterotic String, Nucl. Phys. B 256, 253-284 (1985). https://doi.org/10.1016/0550-3213(85)90394-3

[25] E. W. Kolb, D. Seckel and M. S. Turner, The Shadow World of superstring theories, Nature 314, 415-419 (1985). https://doi.org/10.1038/314415a0

[26] E. Witten, New Issues in Manifolds of SU(3) Holonomy, Nucl. Phys. B 268, 79-112 (1986). https://doi.org/10.1016/0550-3213(86)90202-6

[27] P. Candelas, G. T. Horowitz, A. Strominger and E. Witten, Vacuum Configurations for Superstrings, Nucl. Phys. B 258, 46-74 (1985). https://doi.org/10.1016/0550-3213(85)90602-9

[28] A. Constantin, Y. H. He and A. Lukas, Counting String Theory Standard Models, Phys. Lett. B 792, $258-262$ (2019). https://doi.org/10.1016/j.physletb.2019.03.048

[29] H. W. Braden, P. H. Frampton, T. W. Kephart and A. K. Kshirsagar, Limitations of Heterotic Superstring Phenomenology, 
Phys. Rev. Lett. 56, 2668-2671 (1986). https://doi.org/10.1103/PhysRevLett.56.2668

[30] T. P. Searight, Mirror Matter from a Unified Field Theory, Found. Phys. 51, 11 (2021). https://doi.org/10.1007/s10701-021-00421-4

[31] C. R. Das, L. V. Laperashvili, H. B. Nielsen and A. Tureanu, Mirror World and Superstring-Inspired Hidden Sector of the Universe, Dark Matter and Dark Energy, Phys. Rev. D 84, 063510 (2011). https://doi.org/10.1103/PhysRevD.84.063510

[32] M. Green, J. Schwarz and E. Witten, Superstring Theories (Cambridge University Press, Cambridge, 1989).

[33] M.Yu. Khlopov and K.I. Shibaev New physics from superstring phenomenology, Grav. Cosmol. Suppl. 8N1, 45-52 (2002).

[34] B. Patt and F. Wilczek, Higgs-field portal into hidden sectors, arXiv:hep-ph/0605188 [hep-ph]. https://arxiv.org/abs/hep-ph/0605188

[35] H. Ruegg and M. Ruiz-Altaba, The Stueckelberg field, Int. J. Mod. Phys. A 19 (2004), 3265-3348. https://doi.org/10.1142/S0217751X04019755

[36] S. L. Glashow, Positronium Versus the Mirror Universe, Phys. Lett. B 167, 35-36 (1986). https://doi.org/10.1016/0370-2693(86)90540-X

[37] R. Foot, A. Y. Ignatiev and R. R. Volkas, Physics of mirror photons, Phys. Lett. B 503, 355-361 (2001). https://doi.org/10.1016/S0370-2693(01)00228-3

[38] Y. B. Zeldovich and M. Y. Khlopov, The Neutrino Mass in Elementary Particle Physics and in Big Bang Cosmology, Sov. Phys. Usp. 24, 755-774 (1981). https://doi.org/10.1070/PU1981v024n09ABEH004816

[39] Z. K. Silagadze, Neutrino mass and the mirror universe, Phys. Atom. Nucl. 60, 272-275 (1997). https://arxiv.org/abs/hep-ph/9503481

[40] R. Barbieri, L. J. Hall and K. Harigaya, Minimal Mirror Twin Higgs, JHEP 11, 172 (2016). https://doi.org/10.1016/S0370-2693(01)00228-3

[41] K. Harigaya, R. Mcgehee, H. Murayama and K. Schutz, A predictive mirror twin Higgs with small Z 2 breaking, JHEP 05, 155 (2020). https://doi.org/10.1007/JHEP05(2020) 155

[42] S. Koren and R. McGehee, Freezing-in twin dark matter, Phys. Rev. D 101, 055024 (2020). https://doi.org/10.1103/PhysRevD.101.055024

[43] M. Giannotti, Mirror world and axion: Relaxing cosmological bounds, Int. J. Mod. Phys. A 20, 2454-2458 (2005). https://doi.org/10.1142/S0217751X05024766

[44] Z. Berezhiani, L. Gianfagna and M. Giannotti, Strong CP problem and mirror world: The Weinberg-Wilczek axion revisited, Phys. Lett. B 500, 286-296 (2001). https://doi.org/10.1016/S0370-2693(00)01392-7

[45] V. A. Rubakov, Grand unification and heavy axion, JETP Lett. 65, 621-624 (1997). https://doi.org/10.1134/1.567390

[46] Z. Berezhiani and L. Bento, Neutron - mirror neutron oscillations: How fast might they be?, Phys. Rev. Lett. 96, 081801 (2006). https://doi.org/10.1103/PhysRevLett.96.081801

[47] D. McKeen, M. Pospelov and N. Raj, Smoke and mirrors: Neutron star internal heating constraints on mirror matter, arXiv:2105.09951 [hep-ph]. https://arxiv.org/abs/2105.09951

[48] Z. Berezhiani, R. Biondi, M. Mannarelli and F. Tonelli, Neutron - mirror neutron mixing and neutron stars, [arXiv:2012.15233 [astro-ph.HE]]. https://arxiv.org/abs/2012.15233

[49] Z. Berezhiani, R. Biondi, P. Geltenbort, I. A. Krasnoshchekova, V. E. Varlamov, A. V. Vassiljev and O. M. Zherebtsov, New experimental limits on neutron - mirror neutron oscillations in the presence of mirror magnetic field, Eur. Phys. J. C 78, 717 (2018). https://doi.org/10.1140/epjc/s10052-018-6189-y

[50] C. Abel et al. [nEDM], A search for neutron to mirror-neutron oscillations using the nEDM apparatus at PSI, Phys. Lett. B 812, 135993 (2021). https://doi.org/10.1016/j.physletb.2020.135993

[51] X. Calmet, Hidden Sector and Gravity, Phys. Lett. B 801, 135152 (2020). https://doi.org/10.1016/j.physletb.2019.135152

[52] S. D. McDermott and S. J. Witte, Cosmological evolution of light dark photon dark matter, Phys. Rev. D 101, 063030 (2020). https://doi.org/10.1103/PhysRevD.101.063030

[53] Z. K. Silagadze, Mirror dark matter discovered?, ICFAI U. J. Phys. 2, 143-154 (2009).https://arxiv.org/abs/0808.2595

[54] J. D. Clarke and R. Foot, Mirror dark matter will be confirmed or excluded by XENON1T, Phys. Lett. B 766, 29-34 (2017). https://doi.org/10.1016/j.physletb.2016.12.047

[55] C. Vigo, L. Gerchow, B. Radics, M. Raaijmakers, A. Rubbia and P. Crivelli, New bounds from positronium decays on massless mirror dark photons, Phys. Rev. Lett. 124, 101803 (2020). https://doi.org/10.1103/PhysRevLett.124.101803

[56] A. Caputo, A. J. Millar, C. A. J. O'Hare and E. Vitagliano, Dark photon limits: a cookbook, arXiv:2105.04565 [hep-ph]. https://arxiv.org/abs/2105.04565

[57] R. Foot, Mirror dark matter and the new DAMA/LIBRA results: A Simple explanation for a beautiful experiment, Phys. Rev. D 78, 043529 (2008). https://doi.org/10.1103/PhysRevD.78.043529

[58] A. Addazi, Z. Berezhiani, R. Bernabei, P. Belli, F. Cappella, R. Cerulli and A. Incicchitti, DAMA annual modulation effect and asymmetric mirror matter, Eur. Phys. J. C 75, 400 (2015). https://doi.org/10.1140/epjc/s10052-015-3634-z

[59] R. Cerulli, P. Villar, F. Cappella, R. Bernabei, P. Belli, A. Incicchitti, A. Addazi and Z. Berezhiani, DAMA annual modulation and mirror Dark Matter, Eur. Phys. J. C 77, 83 (2017). https://doi.org/10.1140/epjc/s10052-017-4658-3

[60] R. Foot, DAMA annual modulation from electron recoils, Phys. Lett. B 785, 403-408 (2018). https://doi.org/10.1016/j.physletb.2018.08.070

[61] Z. Berezhiani and A. Lepidi, Cosmological bounds on the 'millicharges' of mirror particles, Phys. Lett. B 681, 276-281 (2009). https://doi.org/10.1016/j.physletb.2009.10.023

[62] P. Ciarcelluti and R. Foot, Early Universe cosmology in the light of the mirror dark matter interpretation of the 
DAMA/Libra signal, Phys. Lett. B 679, 278-281 (2009). https://doi.org/10.1016/j.physletb.2009.07.052

[63] R. Foot and S. Vagnozzi, Solving the small-scale structure puzzles with dissipative dark matter, JCAP 07, 013 (2016). https://doi.org/10.1088/1475-7516/2016/07/013

[64] Z. Berezhiani, P. Ciarcelluti, D. Comelli and F. L. Villante, Structure formation with mirror dark matter: CMB and LSS, Int. J. Mod. Phys. D 14, 107-120 (2005). https://doi.org/10.1142/S0218271805005165

[65] R. Foot and S. Vagnozzi, Dissipative hidden sector dark matter, Phys. Rev. D 91, 023512 (2015). https://doi.org/10.1103/PhysRevD.91.023512

[66] R. Foot and S. Vagnozzi, Diurnal modulation signal from dissipative hidden sector dark matter, Phys. Lett. B 748, 61-66 (2015). https://doi.org/10.1016/j.physletb.2015.06.063

[67] J. L. Synge, Talking About Relativity (North-Holland Pub. Co., Amsterdam, 1970). 